International Journal of Cancer Studies \& Research (IJCR)

ISSN:2167-9118

\title{
The Influence of Lifestyle Risk Factors on the Occurrence of Gynecological Cancers: A Review of the Evidence and Opportunities for Prevention and Management
}

\author{
Review Article
}

Bittoni $\mathrm{MA}^{1 *}$, Fisher $\mathrm{JL}^{2}$, Weier R $\mathrm{R}^{3,4}$

${ }^{1}$ The Ohio State University, College of Medicine, Division of Health Sciences and Medical Dietetics, Columbus, USA.

${ }^{2}$ The Ohio State University, James Cancer Hospital and Solove Research Institute, Columbus, USA.

${ }^{3}$ The Ohio State University, Comprehensive Cancer Center, Columbus, USA.

${ }^{4}$ The Ohio State University, College of Public Health, Division of Epidemiology, Columbus, USA.

\begin{abstract}
Over one million gynecologic cancers have been diagnosed in women worldwide. The five main types of gynecologic cancer are: uterine, ovarian, cervical, vulvar and vaginal. This review describes the current evidence regarding modifiable lifestyle factors and risk of gynecologic cancer, with a focus on the three most prevalent gynecologic cancers: uterine, ovarian, and cervical, which are included among the top seven causes of cancer deaths among women. This review primarily considers more recent evidence (since 2004) regarding the prominent lifestyle factors of obesity, physical activity, diet and smoking and provides a discussion of opportunities for prevention and management of gynecologic cancer. Although smoking and obesity emerged as the strongest risk factors, further research in the form of high quality prospective studies and randomized trials is needed to confirm the results for several risk factors, especially diet and physical activity, and for various populations and subgroups. Women should be educated on how modifiable lifestyle factors may reduce their risk of gynecological cancer. Clinicians should also be made aware of how they can assist patients in implementing behaviors in order to reduce their risk and for survivors to better manage their disease.
\end{abstract}

Keywords: Lifestyle Risk Factors; Smoking; Obesity; Diet; Physical Activity; Endometrial Cancer; Ovarian Cancer; Cervical Cancer.

\section{*Corresponding Author: \\ Marisa A. Bittoni PhD, \\ Tel: 614-206-3518 \\ Fax: 614-459-8798 \\ E-mail: bittoni.1@osu.edu \\ Received: May 16, 2015 \\ Accepted: July 22, 2015 \\ Published: July 23, 2015}

The Ohio State University, College of Medicine, Division of Health Sciences and Medical Dietetics, PO Box10270, Columbus, OH 43210, USA.

Citation: Bittoni MA, Fisher JL, Weier R (2015) The Influence of Lifestyle Risk Factors on the Occurrence of Gynecological Cancers: A Review of the Evidence and Opportunities for Prevention and Management. Int J Cancer Stud Res. S2:001, 1-8. doi: http://dx.doi.org/10.19070/21679118-SI02001

Copyright: Bittoni MA ${ }^{\odot}$ 2015. This is an open-access article distributed under the terms of the Creative Commons Attribution License, which permits unrestricted use, distribution and reproduction in any medium, provided the original author and source are credited.

\section{Introduction}

Gynecologic cancers are caused by uncontrolled growth and spread of abnormal cells originating in the female reproductive organs, including the cervix, ovaries, uterus, fallopian tubes, vagina and vulva. Over one million gynecologic cancers have been diagnosed in women worldwide and almost half have resulted in death [1]. This review describes the current evidence regarding modifiable lifestyle factors and the risk of gynecological cancer, with a focus on the three main types of gynecologic cancer: endometrial, ovarian and cervical, which are among the top seven causes of cancer incidence and deaths among women [2].

\section{Methods}

This focus of this review was limited to the following modifiable lifestyle risk factors of endometrial, ovarian and cervical cancer: obesity, physical activity, diet, and smoking. Risk factors related to medications, medical procedures and parity were not explored for the purpose of this review. In order to review the evidence on lifestyle factors and the risk of gynecological cancer, we conducted a search of the Pubmed/Medline database, which comprises millions of citations from the biomedical literature, life science journals, and online books. Given the broad scope of this review in terms of the number of cancers and risk factors explored, we primarily sought systematic reviews and meta-analysis that were conducted within the last 10 years (since 2004) using keywords that included obesity, physical activity, diet and smoking for endometrial, ovarian and cervical cancers. In some cases, individual studies were considered, especially if no reviews or meta-analysis were available for a specific topic. In order to minimize possible selection bias inherent in using multiple systematic reviews and meta-analysis (resulting in the repeated citation of the same articles), we attempted to restrict the basis of evidence for associations to those not containing overlapping systematic reviews and 
meta-analyses.

\section{Results}

\section{Review and Assessment of the Literature}

Out of over 90 studies that were found that matched the above search criteria, 68 were used for this review. The evidence is presented in the following sections according to the three cancers reported: endometrial, ovarian and cervical. Tables 1-3 summarize the evidence for the association between the various lifestyle factors and endometrial, ovarian and cervical cancer risk, respectively.

\section{Lifestyle Risk Factors and Endometrial Cancer}

Endometrial cancer (i.e. cancer of the lining of the uterus) is the most common gynecological cancer and the fourth most common cancer for women in developed countries, accounting for $6 \%$ of new cancer cases among women in the United States [1, 2]. Lifestyle risk factors also play a prominent role in the etiology and development of endometrial cancer, especially smoking, obesity, diet, and physical activity [3]. Lifestyle factors are often associated with Type I estrogen-dependent endometrial cancer. Type 1 is typically associated with unopposed estrogen, a low-grade histology and an endometroid histologic subtype. Type II, which is non-estrogen dependent and typically occurs in older women, is high grade, and has a serous histologic type $[4,5]$.

Obesity: Epidemiologic studies have consistently shown positive associations between obesity and endometrial cancer risk, which may be explained by alterations in the metabolism of endogenous hormones, production of certain cytokines, adipose-dependent inflammatory reactions, and genetic factors [6]. Obesity is an established risk factor for endometrial cancer, both Type 1 and Type 2 , with an increased risk of approximately $50 \%$ for every 5 -unit increase in body mass index (BMI), especially for women who have never used hormone replacement therapy (HRT) [7, 8]. A recent review and meta-analysis identified 12 published manuscripts on obesity and endometrial cancer survival, 4 of which suggested that obesity assessed by BMI at diagnosis is associated with worse survival among women diagnosed with endometrial cancer, with risk ratios (RR) ranging from 1.86-2.76 for women with a BMI $\geq 40 \mathrm{~kg} / \mathrm{m}^{2}$ compared with non-obese weight women [9]. Obese women with endometrial cancer have also displayed higher mortality, which may be due to obese women being more likely to miss routine cancer screening that results in delayed diagnosis and poorer prognosis [10].

The strongest associations have been observed for adult weight gain and current adiposity, but those who lost weight have also been found to be at decreased risk [11-13]. The World Cancer Research Fund/American Institute for Cancer Research (WCRF/ AICR) 2013 Continuous Update Project rated the strength of the overall evidence on obesity as a causal factor in endometrial cancer development as "convincing" [8].

Physical Activity: Another potentially modifiable risk factor related to obesity is physical activity, which regulates hormonal and metabolic pathways and helps control body weight, and thus may protect against endometrial cancer [14]. Several studies have shown a link between physical activity and endometrial cancer risk. Women who performed $90 \mathrm{~min} /$ day or more of nonoccupational physical activity had decreased endometrial cancer risk (RR, 0.54; 95\% confidence interval (CI), 0.34-.85) compared to those who exercised less than $30 \mathrm{~min} /$ day [15]. A populationbased case control study found that women who reported 7.5 metabolic equivalent (MET)-hours per week had a 34\% lower endometrial cancer risk (OR, 0.66; 95\% CI:0.50-0.87) compared to women who had 0 MET-hours/week, after adjusting for risk factors, including BMI. Also, women who sat $>8$ hours/day had a $52 \%$ increased odds of endometrial cancer (OR, 1.52; $95 \%$ CI:1.07-2.16) compared to those who sat $<4$ hours/day [9]. The European Prospective Investigation into Cancer and Nutrition Study (EPIC), however, found no protective effect of physical activity and endometrial cancer risk, with a limited benefit among postmenopausal women [16].

Table 1. Summary of evidence for associations between lifestyle factors and endometrial cancer risk ${ }^{1}$.

\begin{tabular}{|l|l|}
\hline Factor & Evidence of Association ${ }^{2}$ \\
\hline Obesity & Strong Evidence of Increased Risk \\
\hline Physical Activity & Moderate to Strong Evidence of Increased Risk \\
\hline Diet & Suggestive Evidence, Overall \\
\hline High Fruit and Vegetable Intake & Suggestive Evidence of Mildly to Moderately Decreased Risk \\
\hline High Total Alcohol Intake & Insufficient Evidence, Overall \\
\hline High Intake of Dairy Products & Suggestive Evidence, Overall \\
\hline High Intake of Dietary Fat, Cholesterol and Egg & Suggestive Evidence, Overall \\
\hline High Intake of Coffee & Suggestive Evidence of Mildly to Moderately Decreased Risk \\
\hline High Intake of Green Tea & Suggestive Evidence, Overall \\
\hline High Glycemic Intake & Suggestive Evidence of Mildly to Moderately Decreased Risk \\
\hline High Intake of Isoflavones & Suggestive Evidence of Mildly to Moderately Decreased Risk \\
\hline Tobacco Smoking & Moderate to Strong Evidence of Decreased Risk \\
\cline { 2 - 2 } & Strong Evidence of Decreased Risk Among Postmenopausal Women \\
\hline
\end{tabular}

${ }^{1}$ Table 1 summarizes only associations with endometrial cancer incidence; associations between factors and screening, premalignant lesion risk, and mortality risk are not shown.

${ }^{2}$ Insufficient evidence=lack of studies to make conclusions; Suggestive evidence $=$ some studies support but overall inconsistent; Moderate $=$ evidence supports but some inconsistencies; Strong=overall evidence is consistent and shows strong associations. 
Table 2. Summary of evidence for associations between lifestyle factors and ovarian cancer risk ${ }^{1}$

\begin{tabular}{|l|l|}
\hline Factor & Evidence of Association ${ }^{2}$ \\
\hline Obesity & Limited Evidence, Overall \\
\cline { 2 - 2 } & $\begin{array}{l}\text { Suggestive Evidence of Mildly to Moderately Increased Risk Among Young } \\
\text { Adult and/or Premenopausal Women }\end{array}$ \\
\hline Physical Activity & Suggestive Evidence of Mildly to Moderately Decreased Risk \\
\hline Diet & Insufficient Evidence, Overall \\
\hline High Fruit and Vegetable Intake & Suggestive Evidence of Mildly to Moderately Decreased Risk \\
\hline High Total Alcohol Intake & Suggestive Evidence of Mildly to Moderately Decreased Risk \\
\hline High Intake of Dairy Products & Insufficient Evidence, Overall \\
\hline High Intake of Dietary Fat, \\
Cholesterol and Egg & No Known Association, Overall \\
\hline Tobacco Smoking & Strongly Increases Risk of Mucinous Subtype \\
\cline { 2 - 2 } & $\begin{array}{l}\text { Suggestive Evidence of Mildly to Moderately Increased Risk of Clear Cell } \\
\text { Subtype }\end{array}$ \\
\hline
\end{tabular}

${ }^{1}$ Table 2 summarizes only associations with ovarian cancer incidence; associations between lifestyle factors and screening, premalignant lesion risk, and mortality risk are not shown.

${ }^{2}$ Insufficient evidence=lack of studies to make conclusions; Suggestive evidence $=$ some studies support but overall inconsistent; Moderate $=$ evidence supports but some inconsistencies; Strong=overall evidence is consistent and shows strong associations.

Table 3. Summary of evidence for associations between lifestyle factors and cervical cancer risk ${ }^{1}$

\begin{tabular}{|l|l|}
\hline \multirow{2}{*}{ Factor } & Evidence of Association \\
\hline \multirow{2}{*}{ Physical Activity } & Suggestive Evidence, Overall \\
\cline { 2 - 2 } & Moderately Increases Adenocarcinoma Risk \\
\cline { 2 - 2 } & No Known Association, Squamous Cell Carcinoma Risk \\
\hline Diet & Insufficient Evidence \\
\hline carotene) & Insufficient Evidence, Overall \\
\hline High Intake of Folic Acid & Suggestive Evidence of Moderately Decreased Risk \\
\hline High Intake of Calcium & \\
\hline High Intake of Antioxidants & Suggestive Evidence of Moderately Decreased Risk \\
\hline $\begin{array}{l}\text { High Intake of Long-chain Polyunsatu- } \\
\text { rated Fatty Acids }\end{array}$ & Insufficient Evidence, Overall \\
\hline Multivitamin/Mineral Supplementation & Insufficient Evidence, Overall \\
\hline High Intakes of Fruits and Vegetables & Insufficient Evidence, Overall \\
\hline Tobacco Smoking & Suggestive Evidence of Mildly to Moderately Decreased Risk \\
\hline
\end{tabular}

${ }^{1}$ Table 3 summarizes only associations with cervical cancer incidence; associations between lifestyle factors and screening, premalignant lesion risk, and mortality risk are not shown.

${ }^{2}$ Insufficient evidence $=$ lack of studies to make conclusions; Suggestive evidence $=$ some studies support but very inconsistent; Moderate $=$ evidence supports but inconsistencies remain; Strong $=$ overall evidence is consistent and shows strong associations

Two meta analysis have shown approximately $20-30 \%$ significantly reduced risks of endometrial cancer for the most active versus least active women $[17,18]$. A recent meta-analysis attempted to quantify the dose-response relationship and observed that an increase in leisure-time physical activity may continue to decrease endometrial cancer risk, within the range of 0-50 MET-hours/ week or $0-15$ hours/week [14]. More prospective studies are needed to assess the magnitude of risk reduction and to determine which aspects of physical activity contribute to reduced risk, and during which period of life physical activity is more effective in changing endometrial cancer risk [18]. The WCRF Continuous Update Project rated the overall evidence for physical activity and decreased endometrial cancer risk as "probable" [8].

Diet: The evidence regarding endometrial cancer and diet has not been consistent [10]. Although results from case control studies have shown that foods high in fat and cholesterol (e.g. red meat, margarine and eggs) were positively associated with endometrial cancer, evidence from large prospective studies have not always supported these results, especially in postmenopausal women [10, 19-22]. Evidence on phytoestrogens shows that isoflavones may reduce the risk of endometrial cancer, especially in non-obese women who consume large quantities compared to obese women who consume small amounts of phytoestrogens [23]. Other dietary factors that may decrease endometrial cancer risk include crude and dietary fiber, non-starch polysaccharides, and vitamins 
A and C $[24,25]$. Recent dietary evidence has shown conflicting evidence for vitamin $\mathrm{D}$, with a few case-control studies showing possible associations [26], but a recent large prospective study showing no effect [27]. Dietary fiber, retinol, beta-carotene, vitamin $C$, vitamin $\mathrm{E}$, and vitamin supplementation may decrease the risk of endometrial cancer [28]. The 2013 WCRF/AICR Continuous Update report rated the evidence for most of the above nutrients as "limited-no conclusion" [8].

Decreased risk of endometrial cancer has also been shown for whole grains [24] and fruits and vegetables [29]. One review paper suggested a modest inverse association with vegetable consumption, especially cruciferous vegetables (summary ORs were 0.90 (CI, 0.86-0.95) for total vegetables and 0.79 (CI, 0.69-0.90) for cruciferous vegetables, respectively [29], but two large prospective studies did not support associations between fruit and vegetable consumption and endometrial cancer [29, 30]. More large controlled trials and prospective studies are needed to adequately assess the effects of several dietary factors on endometrial cancer risk [10]. The 2013 WCRF/AICR Project Report for endometrial cancer reported 'sufficient' evidence regarding a probable causal relationship with increasing glycemic load [8]. However, the suggestive inverse association with non-starchy vegetables and positive association with red meat reported in the 2007 report [31] were not confirmed in the 2013 update, which only considered prospective studies.

There has also been considerable interest in the relationship between coffee and tea drinking and cancer risk because tea, especially, is a rich source of antioxidant polyphenols. The 2013 WCRF/AICR report concluded there was now sufficient evidence to suggest a probable inverse relation between coffee consumption and risk of endometrial cancer, but that there was insufficient evidence regarding tea [8]. Je et al. observed a pooled RR of endometrial cancer for the highest versus lowest categories of coffee intake was 0.71 (95\% CI: 0.62-0.81) [32]. A recent prospective study and meta-analysis found no association between tea and endometrial cancer risk, but coffee showed a slight decrease in risk [33]. Although green tea has been associated with reduced risks of endometrial cancer, additional data from prospective studies are needed [34].

Smoking: Smoking has consistently shown an inverse relationship with endometrial cancer risk $[3,35,37]$. A large prospective study recently showed that current and former smokers who recently quit had significantly lower endometrial cancer risks compared to never smokers [38]. A previous meta-analysis of prospective and case-control studies showed that cigarette smoking was associated with a reduced risk of endometrial cancer among current (RR, 0.65; $95 \% \mathrm{CI}, 0.55,0.78)$ and former smokers (RR, 0.89; $95 \%$ CI, 0.80-1.00) compared to never smokers, especially among postmenopausal women [35]. Quitting smoking 1-4 years prior to baseline was significantly associated with endometrial carcinoma risk (RR, 0.65; $95 \% \mathrm{CI}, 0.48-0.89$ ), while quitting 10 or more years before baseline was not [35].

The relative risk of endometrial cancer has been reported to be approximately 0.5 in smokers [39], with a stronger decrease in risk observed in postmenopausal women. In addition, the longer and heavier the duration of smoking, the less the risk for developing endometrial cancer. This association does not appear to be true for passive smokers [40]. The biologic mechanism for these find- ings may be due to the anti-estrogenic effects of smoking [36, 38]. Smokers have shown a lower risk of developing endometrial or clear cell ovarian cancers, a pattern that is consistent with the previously reported inverse association between smoking and risk of endometrial cancer [36]. This somewhat surprising observation has been attributed to the fact that smokers tend to have lower circulating estrogen levels and earlier menopause than nonsmokers [41].

\section{Lifestyle Risk Factors and Ovarian Cancer}

The majority of ovarian cancers either begin in the cells on the surface of the ovary (i.e., epithelial ovarian cancer) or in egg cells (i.e., malignant germ cell tumors). Given the lack of efficacy of ovarian cancer screening, most women are diagnosed at an advanced stage, for which curative treatment is often unavailable [42]. Of the gynecological cancers, epithelial ovarian cancer is the most common cause of cancer-related deaths and the fourth most common cause of cancer-related morbidity among women [42]. Although factors such as family history of or an inherited predisposition (e.g., BRCA1 or BRCA2 mutation) to ovarian cancer increase a woman's risk of developing ovarian cancer that cannot be modified, there are lifestyle risk factors that can potentially be modified to reduce ovarian cancer risk [43].

Obesity: There is limited evidence to suggest obesity is associated with a small to moderately increased risk of ovarian cancer among young adult and/or premenopausal women [44, 45]. Olsen et al. conducted a meta-analysis of 25 studies, for which the pooled effect estimate for obesity versus normal BMI was 1.3 (95\% CI:1.1-1.5) [45]. Using data from five case control studies and four cohort studies that reported on BMI in young adulthood, the pooled effect estimate was 1.22 (95\% CI:1.02-1:45). Of three case-control studies and one cohort study that stratified by menopausal status, three studies reported higher risk estimates associated with elevated BMI among premenopausal women [45]. A pooled analysis of 12 prospective cohort studies did not find a significant association between baseline obesity and ovarian cancer risk among women overall (pooled RR, 1.03; 95\% CI:0.86-1.22) or among women in early adulthood (pooled RR, 1.01; 95\% CI:0.72-1.43) [44]. However, baseline obesity was positively associated with ovarian cancer risk in premenopausal women (pooled RR, 1.72; 95\% CI:1.02-2.89), although the number of premenopausal cases was limited. Both reviews noted that the studies reviewed often lacked sufficient statistical power to detect modest effects by histological subtypes of ovarian cancer and acknowledged limitations in using a single BMI measurement $[44,45]$. Weight change during follow-up may attenuate the risk estimates and Schouten et al. found cases with longer follow-up were more likely to be postmenopausal at diagnosis than those with a shorter follow-up, further suggesting a time-dependent association. The 2013 WCRF/AICR Continuous Update Project rated the evidence for obesity and increased ovarian cancer risk as "probable" [8].

Physical Activity: Some evidence suggests recreational physical activity is associated with a small to moderately decreased risk of ovarian cancer, but research findings regarding this relationship lack consistency [46, 47]. Olsen et al. conducted a meta-analysis of twelve case-control and cohort studies targeting the association between recreational physical activity and histologically confirmed ovarian cancer [46]. For the case control studies, the 
pooled RR for the risk of ovarian cancer associated with highest versus lowest levels of recreational physical activity was 0.79 (95\% CI:0.70-0.85). The pooled RR was 0.81 (95\% CI:0.57-1.17) for the six cohort studies and 0.72 (95\% CI:0.53-0.98) upon exclusion of a study with outlying results. The pooled results of this meta-analysis suggest that recreational physical activity level is inversely associated with ovarian cancer risk [46]. However, in a meta-analysis of cohort and case-control studies on the association between any form of physical activity and ovarian cancer risk, Cust reported one study with a positive association, half observed no association and the remainder reported inverse associations. Among the studies observing an inverse association between physical activity and ovarian cancer risk, dose-response relationships were not consistently found across physical activity categories [47]. For example, Rossing et al. observed a statistically significant inverse dose-response relationship between increasing duration of high-intensity recreational activity and ovarian cancer risk, but not for duration of total recreational physical activity, low-or moderate-intensity activity, or MET-hours per week of recreational activity [48]. Given the inconsistencies in the current literature, additional observational research is needed to elucidate the relationship between different forms of physical activity and ovarian cancer risk. The 2013 WCRF/AICR report rated the evidence on physical activity as "limited-no conclusion" [8].

Diet: The evidence from several reviews of diet and ovarian cancer does not support consistent relationships between moderate intake of dietary factors, including fruit and vegetables, alcohol, dairy products, calcium, fat and cholesterol, and increased risk of ovarian cancer [49-53]. For example, Koushik et al. conducted a pooled analysis of data from 12 prospective studies over 7 to 22 years of follow-up in which fruit and vegetable intake was measured at baseline using a validated food frequency questionnaire (FFQ). The pooled RR for the highest versus the lowest quartile of fruit and vegetable intake was 1.06 (95\% CI:0.92-1.21) and 0.90 (95\% CI:0.78-1.04), respectively, and findings were similar across histologic subtypes [49]. Similarly, Genkinger et al. conducted a pooled analysis of data from 10 prospective cohort studies on 529, 638 women with 2,001 incident epithelial ovarian cases for which the pooled RR comparing $\geq 30$ to $0 \mathrm{~g}$ per day of total alcohol and risk of ovarian cancer was 1.12 (95\% CI:0.86-1.44) [53]. As other reviews on dairy products and ovarian cancer have noted, it is important for future research to take dietary guidelines into account since a lactose intake equivalent to three or more servings of milk per day was associated with a modest increase in ovarian cancer risk, and three servings were recommended by newly released guidelines [52]. One limitation of the prospective studies reviewed is that baseline FFQs tended to capture dietary intake for the year preceding the start of the follow-up period, so it was not possible to account for changes in intake during followup [52]. The $2013 \mathrm{WCRF} / \mathrm{AICR}$ report rated the dietary evidence for ovarian cancer risk as "limited-no conclusion" [8].

Smoking: Although previous research suggested smoking was not associated with an increased risk of ovarian cancer, there is emerging evidence that smoking is positively associated with the mucinous histological subtype of ovarian cancer in a doseresponse manner and that this relationship can be reversed upon smoking cessation [54]. Jordan et al. conducted a systematic literature review and meta-analysis of eight population-based casecontrol studies, one pooled analysis of case-control studies, and one cohort study that included a total of 6,474 persons, 910 of whom had a mucinous ovarian cancer diagnosis, and 5,564 of whom had non-mucinous ovarian cancer diagnosis. Comparing current smokers to never smokers, the risk of mucinous ovarian cancer doubled significantly (pooled RR, 2.1; 95\% CI:1.7-2.7), the risk of serous (pooled RR, 1.0; 95\% CI:0.8-1.2) and endometrioid (pooled RR, 0.8; 95\% CI:0.6-1.1) cancers were similar, and the risk of clear cell ovarian cancers were reduced significantly (pooled RR, 0.6; 95\% CI:0.3-0.9) [54]. Furthermore, although the risk of mucinous cancer increased with increasing smoking levels, it returned to that of never smokers within 20-30 years of smoking cessation [54].

\section{Lifestyle Factors and Cervical Cancer}

Cervical cancer forms in the cervix and usually develops very slowly and without symptoms. Cervical cancer is one of the most preventable cancers, and, therefore, has lower incidence, since it can be identified through regular Pap tests (a procedure in which cervical cells are scraped and microscopically examined) [55]. HPV is the primary etiologic agent of cervical cancer, which almost never occurs without HPV infection. As a result, risk factors for cervical cancer are normally associated with, either directly or indirectly, the process of HPV-associated cervical cancer carcinogenesis [55]. Therefore, it should be noted that each risk factor to be described is potentially associated with HPV transmission, infection, and/or progression to cervical cancer or a precancerous lesion.

Obesity: Results from a review of studies suggest no consistent evidence for the association between obesity and cervical cancer incidence [56]. However, some results suggest a moderately increased cervical adenocarcinoma risk (as opposed to squamous cell carcinoma risk) among obese women [56]. In a matched casecontrol study, Lacey et al. reported that obese women (versus normal weight women) were at increased risk of cervical adenocarcinoma (OR, 2.1; 95\% CI:1.1-3.8) and that women in the highest versus lowest tertile of waist-to-hip ratio were also at increased risk (OR, 1.8; 95\% CI:0.97-3.3), while results pertaining to squamous cell carcinoma were weaker and not statistically significant [57]. Cervical adenocarcinoma, representing approximately 15 percent of cervical cancers, may be more hormonally responsive than squamous cell carcinoma and may exhibit a "mitogenic effect of increased estrogen on glandular cervical cancers." [56] Published studies on possible molecular mechanisms have shown that alterations in the endogenous hormone profiles, production of specific adipose proteins, cytokines and inflammation, and intrinsic genetic factors may play roles in the development and progression of cervical cancer [6]. Cervical cancer mortality risk is also increased among obese women [56, 58-60], which may result from the fact that obese women are less likely to be compliant with HPV screening (i.e. Pap testing) recommendations [56, 59, $61,62]$. Based on a meta-analysis, compared to normal BMI women, OR (95\% CI) for Pap testing were 0.91 (0.80-1.03), 0.81 (0.70$0.93), 0.75(0.64-0.88)$, and $0.62(0.55-0.69)$ for the overweight and class I, II, and III obesity categories, respectively [59]; although, these findings were apparent only for white women [59, 60]. Another literature review suggested an inverse association between decreased Pap testing and increasing body size, and, again, several studies reported significant associations among white women only [61]. The 2007 WCRF/AICR report rated the evidence on 
obesity as "limited-no conclusion" [8].

Physical Activity: There is currently insufficient evidence concerning the potential role of physical activity in the development of cervical cancer $[47,58]$. It is possible that physical activity impacts cervical intraepithelial neoplasias (CIN) or cervical carcinogenesis through hormonal and/or immune function but few studies have been conducted. Lee et al. [63] found that, compared to women with lower levels of leisure-time physical activity $(<38.5$ MET-hours/week), women with higher levels (>73 MET hours/ week) had significantly lower risk of both CIN2/3 (OR, 0.58; 95\% CI:0.36-0.93) and invasive cervical cancer (OR, 0.61; 95\% CI:0.38-0.98), even after adjustment for other factors. Additional studies are needed to examine the relationship between physical activity and cervical cancer risk. No ratings have been provided for physical activity from the 2007 or 2013 WCRF/AICR reports.

Diet: Overall, there are few consistent associations between dietary factors and cervical cancer risk from large, well-designed observational studies or randomized controlled trials. Dietary factors may serve as cofactors in cervical carcinogenesis, but the evidence is inconclusive [31]. Because HPV infection is a necessary cause of cervical cancer and since CIN is a premalignant lesion in the natural history of cervical cancer, Chih et al. reviewed studies of the dietary prevention of both HPV infection and CIN, reporting that high levels of dietary intakes of fruits and vegetables protect against CIN, and dietary intakes of some nutrients (especially those with high antiviral and antioxidant potential) may prevent progression of transient and persistent HPV infections to high-grade CIN/in situ cervical cancer [64]. They report that high intake of "retinol and vitamin A, bioavailable calcium, antioxidants (including vitamin C, E, carotene, lutein and lycopene), as well as long-chain polyunsaturated fatty acids can significantly reduce the risk of in situ cervical cancer" [64]. Results from a large European prospective study of almost 300,000 women, suggested a statistically significant inverse association of invasive squamous cervical cancer with a daily increase in intake of 100 grams of total fruits (HR, 0.83; 95\% CI:0.72-0.98) and non-significant associations with total vegetables and most other foods, including beta-carotene, vitamin $\mathrm{D}$, and folic acid (which had each been suggested as protective from previous studies) [65]. In a metaanalysis of 22 case-control studies and over 10,000 participants, Myung et al. reported statistically significant associations between cervical neoplasia and intakes of vitamin B12 (RR, 0.35; 95\% CI:0.19-0.63), vitamin C (RR, 0.67; 95\% CI:0.55-0.82), vitamin E (RR, 0.56; 95\% CI:0.35-0.88), and beta-carotene (RR, 0.68; 95\% CI:0.55-0.84), while for multivitamin and mineral supplements, no reduction in cancer incidence was observed [66]. Results from another meta-analysis based on 12,000 participants revealed associations between cervical cancer risk and dietary intakes of retinol (OR, 0.80; 95\% CI:0.64-1.00), carotene (OR, 0.51; 95\% CI:0.350.73 ) and other carotenoids (OR, 0.60; 95\% CI:0.43-0.84) [67]. While some evidence exists for fruits and vegetables and the complex relationship between folic acid, its metabolism and cervical cancer carcinogenesis, further study is warranted due to the lack of consistency of the evidence. The WCRF/AICR 2007 report rated carrots as having "probable" evidence of decreased ovarian cancer risk, but non-starchy vegetables, fruit, milk, retinol and vitamin E were all rated as "limited- no conclusion" [8].

Smoking: Smoking increases cervical cancer risk, although the mechanism in cervical carcinogenesis is not fully understood. Among HPV-infected women, current and former smokers have approximately two to three times the risk of high-grade CIN or invasive cancer [68]. Current smoking increases squamous, but not adenocarcinoma, cervical cancer risk up to three-fold with increasing cigarettes smoked per day [69].

\section{Discussion}

The evidence cited in this review confirms that several modifiable lifestyle risk factors play a prominent role in the development of endometrial, cervical and ovarian cancer. In particular, the risk factors of obesity, physical activity, and tobacco smoking have been shown to affect each of these cancers differentially. Although few of these factors have been identified as demonstrating conclusive evidence of increased or decreased risk, such as obesity and increased endometrial cancer risk, it is believed that enough evidence has been shown to provide suggestions for prevention and management of these cancers, as described in the following section.

\section{Opportunities for Prevention and Management}

As shown in Tables 1-3, smoking is strongly associated with ovarian and cervical cancer risk (in addition to many other cancers), but protective of endometrial cancer risk, although it decreases survival and results in worse outcomes $[3,70]$. Due to its overall adverse effects on cancer risk and mortality, a reduction in the prevalence of smoking should be a public health priority. Although smoking rates have declined in the last decade, more efforts should be made to further reduce them.

Obesity showed the strongest evidence for increased endometrial cancer risk, but only showed positive associations for selected subgroups for ovarian cancer, and selected cancer subtypes for cervical cancer. Given that obesity is also associated with reduced survival for many cancers, the reduction of obesity is another primary target for cancer prevention and management $[6,70]$.

Although dietary factors and physical activity varied the most with regards to cancer risk for the various gynecological cancers, it is well known that a healthy diet and increased physical activity are integral for reducing obesity, and, therefore, individuals should be encouraged to adopt these behaviors, especially for reducing endometrial cancer risk, which appeared to have the strongest association.

In addition, lifestyle risk factors may impact the success of treatment, mortality risk, and survival probability of gynecological cancers. Indeed, for example, endometrial and cervical cancer survivors who are obese $(\mathrm{BMI}>30)$ and use tobacco have reduced probabilities of survival [6]. It is likely that other deleterious health factors may directly impact survival probability, although more research is needed in this area.

\section{Limitations}

This review primarily summarized systematic reviews and metaanalysis conducted within the last 10 years (since 2004) regarding the association between modifiable lifestyle risk factors and uterine, ovarian, and cervical; individual studies were cited when the evidence for a specific topic was sparse. This approach was necessary due to the broad scope of this topic, and although it was an efficient way to gather the most up-to-date evidence, the 
review of "systematic reviews and meta-analysis" can amplify selection bias inherent in the published meta-analysis and systematic reviews. In addition, some of the early and/or key findings may have been given greater weight in this manuscript because they could have been included in several different reviews and/ or meta-analysis, although we sought to include non-overlapping reviews and to cite individual studies only when reviews or metaanalysis were not available.

\section{Strengths}

One of the primary strengths of this study is that it synthesizes the more recent evidence (within the last 10 years) on modifiable lifestyle risk factors and endometrial, cervical and ovarian cancer. The paper also provides a discussion of several strategies for the prevention and management of gynecological cancer based on the information obtained from this review, which can be used to help develop and target prevention programs for reducing these gynecologic cancers.

\section{Conclusion}

Lifestyle risk factors play a prominent role in the development of gynecological cancers and may also impact disease progression and survival. Although smoking and obesity emerged as the strongest risk factors, further research in the form of high quality prospective studies and randomized trials is needed to confirm the results for several risk factors, especially diet and physical activity, and for various populations and subgroups. Women should be educated on how modifiable lifestyle factors and associated behaviors may reduce their risk of several gynecological cancers. Clinicians should also be made aware of how they can assist patients in implementing risk-reducing behaviors and help survivors better manage their diseases.

\section{References}

[1]. Siegel RL, Miller KD, Jemal A (2015) Cancer statistics, 2015. CA Cancer J Clin 65(1): 5-29.

[2]. Ferlay J, Soerjomataram I, Ervik M, et al. GLOBOCAN 2012 v1.0, Cancer Incidence and Mortality Worldwide: IARC Cancer Base No. 11 [Internet]. Lyon, France, International Agency for Research on Cancer, 2013. http:// globocan.iarc.fr.

[3]. Holman L, Lu K (2012) The epidemiology of endometrial cancer. Global Library of Women's Medicine[serial online]. Available from URL: http:// www.glowm.com/section_view/heading/The Epidemiology of Endometrial Cancer/item/236 - r8.

[4]. Setiawan VW, Yang HP, Pike MC, McCann SE, Yu H, et al. (2013) Type I and II Endometrial Cancers: Have They Different Risk Factors? J Clin Oncol 31(20): 2607-2618.

[5]. Davoodi SH, Malek-Shababi T, Moghadam A, Shahbazi R, Esmaeili S (2013) Obesity as an Important Risk Factor for Certain Types of Cancer. Iran J Cancer Prev 6(4): 186-194.

[6]. Gu W, Chen C, Zhao KN (2013) Obesity-associated endometrial and cervical cancers. Front Biosci (Elite Edition) 5: 109-118.

[7]. Crosbie EJ, Zwahlen M, Kitchener HC, Egger M, Renehan AG (2010) Body mass index, hormone replacement therapy and endometrial cancer risk: a meta-analysis. Cancer Epidemiol Biomarkers Prev 19(12): 3119-3130.

[8]. World Cancer Research Fund (WCRF)/American Institute for Cancer Research (AICR). (2013) Continuous Update Project Report: Food, Nutrition, Physical Activity and the Prevention of Endometrial Cancer: http://www. dietandcancerreport.org.

[9]. Arem H, Irwin M (2013) Obesity and endometrial cancer survival: a systematic review. Int J Obes 37(5): 634-639.

[10]. Linkov F, Edwards R, Balk J, Yurkovetsky Z, Stadterman B, et al. (2008) Endometrial hyperplasia, endometrial cancer and prevention: Gaps in exisiting research of modifiable risk factors. Eur J Cancer 44(12): 1632-1644.

[11]. Nagle CM, Marquart L, Bain CJ, O'Brien S, Lahmann PH, et al. (2013)
Impact of weight change and weight cycling on risk of different subtypes of endometrial cancer. Eur J Cancer 49(12): 2717-2726.

[12]. Yang HP, Wentzensen N, Tabert B, Gierach GL, Felix AS, et al. (2012) Endometrial cancer risk factors by 2 main histologic subtypes: the NIH-AARP Diet and Health Study. Am J Epidemiol 177(2): 142-151.

[13]. Chang SC, Lacey JV Jr, Brinton LA, Hartge P, Adams K, et al. (2007) Lifetime weight history and endometrial cancer risk by type of menopausal hormone use in the NIH-AARP diet and health study. Cancer Epidemiol Biomarkers Prev 16(4): 723-730.

[14]. Keum N, Ju W, Lee DH, Ding EL, Hsieh CC, et al. (2014) Leisure-time physical activity and endometrial cancer risk: dose-response meta-analysis of epidemiological studies. Int J Cancer 135(3): 682-694.

[15]. Schouten LJ, Goldbohm RA, Van Den Brandt PA (2004) Anthropometric, physical activity, and endometrial cancer risk: results from the Netherlands Cohort Study. J Natl Cancer Inst 96(21): 1635-1638.

[16]. Freidenreich C, Cust A, Lahmann P, Steindorf K, Boutron-Ruault MC, et al. (2007) Physical activity and risk of endometrial cancer: the European prospective investigation into cancer and nutrition. Int J Cancer 121(2): 347-355.

[17]. Moore S, Gierach G, Schatzkin A, Matthews CE (2010) Physical activity, sedentary behaviours, and the prevention of endometrial cancer. Br J Cancer 103(7): 933-938.

[18]. Voskuil D, Monninkhof E, Elias S, Vlems FA, van Leeuwen FE (2007) Physical activity and endometrial cancer risk, a systematic review of current evidence. Cancer Epidemiol Biomarkers Prev 16(4): 639-648.

[19]. Genkinger JM, Friberg E, Goldbohm RA, Wolk A (2012) Long-term dietary heme iron and red meat intake in relation to endometrial cancer risk. Am J Clin Nutr 96(4): 848-854.

[20]. Di Maso M, Talamini R, Bosetti C, Montella M, Zucchetto A, et al. (2013) Red meat and cancer risk in a network of case-control studies focusing on cooking practices. Ann Oncol 24(12): 3107-3112.

[21]. Biel RK, Csizmadi I, Cook LS, Courneya KS, Magliocco AM, et al. (2011) Risk of endometrial cancer in relation to individual nutrients from diet and supplements. Public Health Nutr 14(11): 1948-1960.

[22]. Biel RK, Friedenreich CM, Csizmadi I, Robson PJ, McLaren L, et al. (2011) Case-control study of dietary patterns and endometrial cancer risk. Nutr Cancer 63(5): 673-686.

[23]. Neill AS, Ibiebele T, Lahmann PH, Hughes MC, Nagle CM, et al. (2014) Dietary phyto-oestrogens and the risk of ovarian and endometrial cancers: findings from two Australian case-control studies. Br J Nutr 111(8): 14301440.

[24]. Aarestrup J, Kyrø C, Christensen J, Kristensen M, Würtz AM, et al. (2012) Whole grain, dietary fiber, and incidence of endometrial cancer in a Danish cohort study. Nutr Cancer 64(8): 1160-1168.

[25]. Bidoli E, Pelucchi C, Zucchetto A, Negri E, Dal Maso L, et al. (2010) Fiber intake and endometrial cancer risk. Acta Oncol 49(4): 441-446.

[26]. McCullough ML, Bandera EV, Moore DF, Kushi LH (2008) Vitamin D and calcium intake in relation to risk of endometrial cancer: a systematic review of the literature. Prev Med 46(4): 298-302.

[27]. Liu JJ, Bertrand KA, Karageorgi S, Giovannucci E, Hankinson SE, et al. (2013) Prospective analysis of vitamin D and endometrial cancer risk. Ann Oncol 24(3): 687-692.

[28]. Xu WH, Dai Q, Xiang YB, Zhao GM, Ruan ZX, et al. (2007) Nutritional factors in relation to endometrial cancer: a report from a population-based case-control study in Shanghai, China. Int J Cancer 120(8): 1778-1781.

[29]. Kabat GC, Park Y, Hollenbeck AR, Schatzkin A, Rohan TE (2010) Intake of fruits and vegetables, and risk of endometrial cancer in the NIH-AARP Diet and Health Study. Cancer Epidemiol 34(5): 568-573.

[30]. McCullough ML, Bandera EV, Patel R, Patel AV, Gansler T, et al. (2007) Kushi LH, Thun MJ, Calle EE. A prospective study of fruits, vegetables, and risk of endometrial cancer. Am J Epidemiol 166(8): 902-911.

[31]. World Cancer Research Fund (WCRF) /American Institute for Cancer Research (AICR). (2007) Food, Nutrition, Physical Activity, and the Prevention of Cancer: a Global Perspective. Washington DC: AICR.

[32]. Je Y, Giovannucci E (2012) Coffee consumption and risk of endometrial cancer: findings from a large up-to-date meta-analysis. Int J Cancer 131(7): 1700-1710.

[33]. Yang TO, Crowe F, Cairns BJ, Reeves GK, Beral V (2015) Tea and coffee and risk of endometrial cancer: cohort study and meta-analysis. Am J Clin Nutr 101(3): 570-578.

[34]. Kakuta Y, Nakaya N, Nagase S, Fujita M, Koizumi T, et al. (2009) Case-control study of green tea consumption and the risk of endometrial endometroid adenocarcinoma. Cancer Causes Control 20(5): 617-624.

[35]. Felix AS, Yang HP, Gierach GL, Park Y, Brinton LA (2014) Cigarette smoking and endometrial carcinoma risk: the role of effect modification and tumor heterogeneity. Cancer Causes Control 25(4): 479-489.

[36]. Zhou B, Yang L, Sun Q, Cong R, Gu H, et al. (2008) Cigarette smoking and the risk of endometrial cancer: a meta-analysis. Am J Med 121(6): 501-508. 
[37]. US Dept of Health and Human Services. (2006) The health consequences of involuntary exposure to tobacco smoke: A Report of the Surgeon General. Atlanta, GA.

[38]. Land SR, Liu Q, Wickerham DL, Costantino JP, Ganz PA (2014) Cigarette smoking, physical activity, and alcohol consumption as predictors of cancer incidence among women at high risk of breast cancer in the NSABP P-1 trial. Cancer Epidemiol Biomarkers Prev 23(5): 823-832.

[39]. Brinton LA, Lacey J, Sherman ME, Sherman VM (2005) Epidemiology of Gynecologic Cancers, in Principles and Practice of Gynecologic Oncology. (4th edtn), Lippincott Williams \& Wilkins, Philadelphia. 3-9.

[40]. Yang HP, Brinton LA, Platz EA, Lissowska J, Lacey JV Jr, et al. (2010) Active and passive cigarette smoking and the risk of endometrial cancer in Poland. Eur J Cancer 46(4): 690-696.

[41]. Tanko LB, Christiansen C (2004) An update on the antiestrogenic effect of smoking: a literature review with implications for researchers and practitioners. Menopause 11(1): 104-109.

[42]. Jayson GC, Kohn EC, Kitchener HC, Ledermann JA (2014) Ovarian Cancer. The Lancet 384(9951): 1376-1388.

[43]. National Cancer Institute: $\mathrm{PDQ}^{\circ}$ Ovarian Cancer Prevention. Bethesda, MD: National Cancer Institute. Date last modified 02/06/2015. Available at:http://cancer.gov/cancertopics/pdq/prevention/ovarian/HealthProfessional.

[44]. Schouten LJ, Rivera C, Hunter DJ, Spiegelman D, Adami HO, et al. (2008) Height, body mass index, and ovarian cancer: a pooled analysis of 12 cohort studies. Cancer Epidemiol Biomarkers Prev 17(4): 902-912.

[45]. Olsen CM, Green AC, Whiteman DC, Sadeghi S, Kolahdooz F, et al. (2007) Obesity and the risk of epithelial ovarian cancer: a systematic review and meta-analysis. Eur J Cancer 43(4): 690-709.

[46]. Olsen CM, Bain CJ, Jordan SJ, Nagle CM, Green AC, et al. (2007) Recreational physical activity and epithelial ovarian cancer: a case-control study, systematic review, and meta-analysis. Cancer Epidemiol Biomarkers Prev 16(11): 2321-2330.

[47]. Cust AE (2011) Physical activity and gynecologic cancer prevention. Recent Results Cancer Res 186: 159-185.

[48]. Rossing MA, Cushing-Haugen KL, Wicklund KG, Doherty JA, Weiss NS (2010) Recreational physical activity and risk of epithelial ovarian cancer. Cancer Causes Control 21(4): 485-491.

[49]. Koushik A, Hunter DJ, Spiegelman D, Anderson KE, Arslan AA, et al. (2005) Fruits and vegetables and ovarian cancer risk in a pooled analysis of 12 cohort studies. Cancer Epidemiol Biomarkers Prev 14(9): 2160-2167.

[50]. Crane TE, Khulpateea BR, Alberts DS, Basen-Engquist K, Thomson CA (2014) Dietary Intake and Ovarian Cancer Risk: A Systematic Review. Cancer Epidemiol Biomarkers Prev 23(2): 255-273.

[51]. Genkinger JM, Hunter DJ, Spiegelman D, Anderson KE, Beeson WL, et al. (2006) A pooled analysis of 12 cohort studies of dietary fat, cholesterol and egg intake and ovarian cancer. Cancer Causes Control 17(3): 273-285.

[52]. Genkinger JM, Hunter DJ, Spiegelman D, Anderson KE, Arslan A, et al. (2006) Dairy products and ovarian cancer: a pooled analysis of 12 cohort studies. Cancer Epidemiol Biomarkers Prev 15(2): 364-372.

[53]. Genkinger JM, Hunter DJ, Spiegelman D, Anderson KE, Buring JE, et al. (2006) Alcohol intake and ovarian cancer risk: a pooled analysis of 10 cohort studies. Br J Cancer 94(5): 757-762.

[54]. Jordan SJ, Whiteman DC, Purdie DM, Green AC, Webb PM (2006) Does smoking increase risk of ovarian cancer? A systematic review. Gynecol Oncol 103(3): 1122-1129.

[55]. National Cancer Institute: What You Need to Know About Cervical Cancer. Bethesda, MD: National Cancer Institute. Date last modified January 2012. Available at: http://www.cancer.gov/publications/patient-education/cervix. pdf.

[56]. Modesitt SC, van Nagell JR Jr (2005) The impact of obesity on the incidence and treatment of gynecologic cancers: a review. Obstet Gynecol Surv 60(10): 683-692.

[57]. Lacey JV Jr, Swanson CA, Brinton LA, Altekruse SF, Barnes WA, et al. (2003) Obesity as a potential risk factor for adenocarcinomas and squamous cell carcinomas of the uterine cervix. Cancer 98(4): 814-821.

[58]. Rieck G, Fiander A (2006) The effect of lifestyle factors on gynaecological cancer. Best Pract Res Clin Obstet Gynaecol 20(2): 227-251.

[59]. Maruthur NM, Bolen SD, Brancati FL, Clark JM (2009) The association of obesity and cervical cancer screening: a systematic review and meta-analysis. Obesity (Silver Spring) 17(2): 375-381.

[60]. Aldrich T, Hackley B (2010) The impact of obesity on gynecologic cancer screening: an integrative literature review. J Midwifery Women's Health 55(4): 344-356.

[61]. Cohen SS, Palmieri RT, Nyante SJ, Koralek DO, Kim S, et al. (2008) Obesity and screening for breast, cervical, and colorectal cancer in women: a review. Cancer 112(9): 1892-1904.

62]. Branković I, Verdonk P, Klinge I (2013) Applying a gender lens on human papillomavirus infection: cervical cancer screening, HPV DNA testing, and HPV vaccination. Int J Equity Health 12(14): 1-10.

[63]. Lee JK, So KA, Piyathilake CJ, Kim MK (2013) Mild Obesity, Physical Activity, Calorie Intake, and the Risks of Cervical Intraepithelial Neoplasia and Cervical Cancer. PLoS ONE 8(6): 1-8.

[64]. Chih HJ, Lee AH, Colville L, Binns CW, Xu D (2013) A review of dietary prevention of human papillomavirus-related infection of the cervix and cervical intraepithelial neoplasia. Nutr Cancer 65(3): 317-328.

[65]. González CA, Travier N, Luján-Barroso L, Castellsagué X, Bosch FX, et al. (2011) Dietary factors and in situ and invasive cervical cancer risk in the European prospective investigation into cancer and nutrition study. Int J Cancer 129(2): 449-459.

[66]. Myung SK, Ju W, Kim SC, Kim H (2011) Vitamin or antioxidant intake (or serum level) and risk of cervical neoplasm: a meta-analysis. BJOG: An International Journal Of Obstetrics And Gynaecology 118(11): 1285-1291.

[67]. Zhang X, Dai B, Zhang B, Wang Z (2012) Vitamin A and risk of cervical cancer: a meta-analysis. Gynecol Oncol 124(2): 366-373.

[68]. Bosch FX, de Sanjosé S (2007) The epidemiology of human papillomavirus infection and cervical cancer. Dis Markers 23(4): 213-227.

[69]. Harper DM, Demars LR (2014) Primary strategies for HPV infection and cervical cancer prevention. Clin Obstet Gynecol 57(2): 256-278.

[70]. Schlumbrecht MP, Sun CC, Huang MS, Zandstra F, Bodurka DC (2014) Lifestyle modification in cervical cancer survivors: an ongoing need. Int J Gynecol Cancer 24(3): 570-575. 